EVS26

Los Angeles, California, May 6-9, 2012

\title{
The North East England Electric Vehicle and Infrastructure Trials
}

\author{
Prof. Phil Blythe ${ }^{1}$, Dr Graham Hill ${ }^{1}$, Dr Yvonne Huebner ${ }^{1}$, Visalakshmi Suresh ${ }^{1}$, John \\ Austin $^{2}$, Liz Gray ${ }^{2}$ and Josey Wardle ${ }^{3}$ \\ ${ }^{I}$ Transport Operations Research Group, Newcastle University, School of Civil Engineering and Geoscience, \\ Newcastle upon Tyne, NE1 7RU, United Kingdom, Phil.Blythe@ncl.ac.uk \\ ${ }^{2}$ Future Transport Systems Ltd., Milburn House, Dean Street, Newcastle upon Tyne, NE1 ILE \\ ${ }^{3}$ Low Carbon Futures Ltd
}

\begin{abstract}
This paper will provide an update and overview of the progress with the North East of England trials in the UK and provide an insight into how early adopters are using their vehicle and importantly their charging behaviour with a large number of charging points available to them.
\end{abstract}

The North East of England is one of the pioneers in the wide-scale demonstration of electric vehicles in the United Kingdom. The SwitchEV trial is one of only 8 projects across the UK to have won funding through the Technology Strategy Board's Ultra Low Carbon Vehicle (ULCV) Demonstrator Programme. As part of the three year trial, 44 full-electric vehicles have been equipped with data loggers to provide an insight into how early adopters are using their vehicles and most importantly their charging behaviour with a large number of charging points available to them. The trials correlates the driving and performance data derived from the vehicles, with charging behaviour derived from both the vehicle loggers and EV recharging infrastructure and soft data from: questionnaires; focus groups; and structured interviews with participants.

Keywords: Electric Vehicle, Charging Infrastructure, data loggers, North East England trials, Switch-E Project

\section{Introduction}

The North East of England is one of the pioneers in the wide-scale demonstration of electric vehicles in the United Kingdom. The SwitchEV trial is one of only 8 projects across the UK to have won funding through the TSB's (Technology Strategy Board), Ultra Low Carbon Vehicle (ULCV) Demonstrator Programme. As part of the three year trial, 44 all electric vehicles have been equipped with data loggers to provide an insight into how early adopters are using their vehicles and most importantly their charging behaviour with a large number of charging points available to them.

\subsection{The Switch EV Trial}

The North East's Switch EV electric vehicle trial is one of eight national trials of electric \& hybrid vehicles funded by the Technology Strategy Board’s (TSB's) £25m Ultra Low Carbon Vehicle Demonstrator Programme [1]. This programme is part of the national strategy to reduce carbon emissions from car and light commercial vehicle use which currently makes up $13 \%$ of the UK's carbon emissions. The programme's primary objective is to understand what barriers there are to 
mass adoption of ultra-low carbon vehicles such as electric cars. SwitchEV is a consortium led by Nissan which sees Newcastle University, AVID vehicles, Liberty Electric Cars, Smith Electric Vehicles and Simon Bailes Peugeot working together to deliver 44 new and innovative fullelectric vehicles onto the roads of the North East. The trial started in November 2010 and extends until May 2013. In that time members of the general public are invited to use the vehicles for 6 months periods as they would their conventional petrol or diesel powered cars (to date we have had 3 cohorts of drivers) [2].

The trial is somewhat unique nationally in that it is operating in conjunction with the Charge Your Car 'Plugged in Places' project This is installing publically accessible charging infrastructure for EV drivers throughout the region. Drivers of the SwitchEV vehicles will now have real options about when and where they charge up. Their homes can be equipped with the latest domestic charging units, they can also have access to the on street charging posts or take advantage of the fast chargers being installed around the region. These enable the cars to be $80 \%$ charged in about 20 minutes. This infrastructure environment enables the research team at Newcastle University to confirm the performance and suitability of the vehicles and infrastructure, and understand the perceptions of the drivers over their 6 months experience of the vehicles.

The Switch EV research team have equipped all the vehicles with loggers that track the vehicles and how they are driven 24 hours a day. They also track where the cars are charged and how much energy they are consuming. The team will also be meeting with the drivers to understand their experiences with the cars and judge their suitability and the potential barriers to their mass adoption [3]. The findings from the trial will be shared with the TSB to help them build the national picture and will also be used by the consortium partners to confirm \& improve their EV offering.

The vehicles in this trial are:

- 6 Avid CUE-V

- 2 Liberty Electric Cars E-Range

- 15 Nissan LEAF

- 20 Peugeot iOn

- 1 Smith Electric Vehicle Edison Minibus

\subsection{Charge Your Car Infrastructure}

Charge Your Car (CYC) is the scheme brand for the Plugged in Places project for the North East of England, funded with $£ 7.8 \mathrm{~m}$ by the Office for Low Emission Vehicles, One North East and regional partners. Its aim is to create a comprehensive connected network of electric vehicle charging points throughout North East of England by March 2013. To date over 300 charge points have been installed in public, commercial and workplace locations with a further 80 domestic charging units in use at the homes of EV drivers in the region. A growing network of eight $50 \mathrm{~kW}$ DC Quick Chargers along the main arterial routes is also providing an $80 \%$ charge in 30 minutes. The Charge Your Car back office and EV User Membership Scheme has been successfully supporting EV Drivers and operators of charging infrastructure since October 2010, providing a single access tool and live charge point status map Fig 1. The Charge Your Car team has also been working with national and international organisations to develop common standards for EV charging technology and to evaluate different charging technologies. Working alongside the SwitchEV project has enabled data from both trials to be correlated together to collectively study EV user's charging behaviour and act upon feedback, to inform future infrastructure developments and strategies [4].

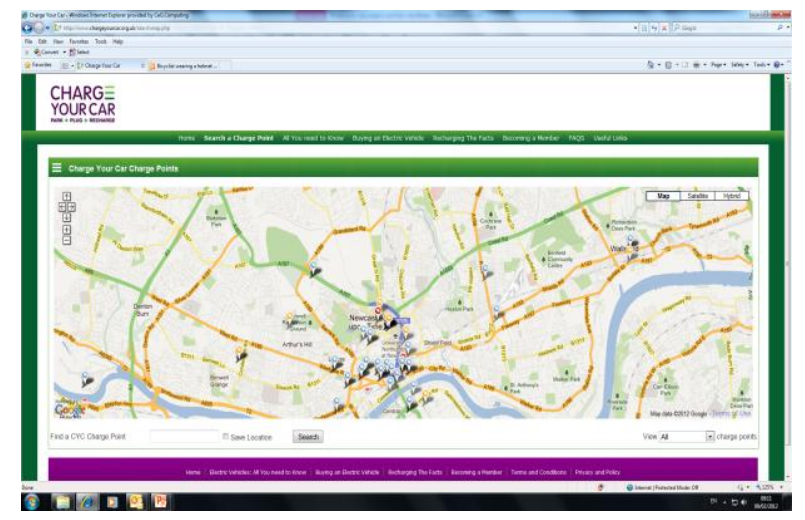

Figure 1: Charge Your Car Webmap of charging point availability in central Newcastle upon Tyne

The key elements of the North East Electric Vehicle activity is centred around 44 electric vehicles being trialled under the TSB ULCV programme. The vehicles are fitted with data loggers that provide a range of driving and vehicle performance data as well as GPS and a time stamp. These data are collected and analysed at Newcastle University. In parallel with the 'hard' data 
collection, questionnaires and focus groups are used to gain an insight into the drivers views and attitudes to electric vehicles, eco-driving and other related issues, before, during and after their trial with electric vehicles (which typically can be from a 3 month period to 2 years). These two sets of data are correlated to explore trends, changes in driving (and re-charging behaviour) and attitudes to electric vehicles, charging and key issues such as cost and 'range anxiety'. The third strand of the NE Electric Vehicle strategy is the provision of a widespread re-charging and associated ITS infrastructure. Which is largely funded through OLEV's ${ }^{i}$ Plugged in Places (PIP) project. To date 307 charging points have been installed across the North East with another 600 scheduled by the end of 2012 . The data on when the units are used, by whom and for how long is again correlated with the two previous sets of data.

This wealth of data is enabling the North East to be pioneering in the understanding of the practical use of electric vehicles, how users perceive them, the barriers that there may be to users regarding them as fit for purpose and an understanding of charging behaviour and concerns. Strategically this enables the NE team to better understand what information and services driver may require and also helps to pinpoint practical and effective locations for future charge points. Moreover the fact the NE trial will be the only one where a large number of charging points are in place at the start of the trail - the real-life behaviour of EV users can be charted and analysed.

\section{Data Collection}

Data is collected from three sources in the trial:

- In-vehicle data (hard data);

- Data from triallists and stakeholders (soft data); and

- Charging data (from the CYC infrastructure).

\subsection{In-Vehicle Data}

The greatest technical challenge has been the collection of real-time data from vehicle. To facilitate this a data logger was developed for use in this and other eco-driving projects. The logger connects to the CAN bus through the vehicles OBD (on-board diagnostics port). The logger has been designed to take some external analogue and digital inputs. These inputs include the GPS and time-stamp data as well as a number of analogue inputs from current-clamps which are attached to various electrical systems of the vehicle to measure current flow and battery drain (and regeneration). The basic data collected is described in table 1.

Data is continuous but the variables measured and the rate of logging is dependent on the state of the vehicle's ignition. With the ignition on the measurement takes place every second, this measurement rate drops to every minute when the ignition is off. This is because when the ignition is off the only information available is charging information through the AC supply and the slow drain of current needed to keep the battery warm. Neither of these two variables are expected to vary much in the 1 second regime, hence 1 minute is enough to capture all features of interest. However the measurement is currently limited to $1 \mathrm{~Hz}$ with ignition on to reduce the amount of data which needs to be transferred.

Table 1 the different variables recorded in ignition 'on' and 'off' modes

\begin{tabular}{|l|l|l|}
\hline Variable & Ignition Off & Ignition On \\
\hline GPS position & & $\checkmark$ \\
\hline Time/Date & $\checkmark$ & $\checkmark$ \\
\hline AC Supply Connection & $\checkmark$ & \\
\hline AC Heater Request & $\checkmark$ & \\
\hline AC Heater State & $\checkmark$ & \\
\hline HV Battery Current & $\checkmark$ & $\checkmark$ \\
\hline HV Battery Voltage & & $\checkmark$ \\
\hline HV Battery Depth of Discharge & & $\checkmark$ \\
\hline Brake Pedal Pressed & & $\checkmark$ \\
\hline Light On & & $\checkmark$ \\
\hline Rear Window Heater & & $\checkmark$ \\
\hline Outside Temperature & & $\checkmark$ \\
\hline
\end{tabular}

The amount of data generated with ignition on is high and this does impose significant bandwidth requirements when being transferred by GPRS to the University server. Tests were undertaken to investigate where a lower sampling rate could be utilised - the results suggested this was possible for the specific data the researchers were contracted to deliver from the trial to the TSB, however for more in-depth analysis of the trial data off-line it was deemed prudent to keep the sample rate at $1 \mathrm{~Hz}$ to record the richness of the available data.

Due to the commercial sensitivity of the decoding of specific OEMs data from their CAN-Bus system 
routines for data interpretation and analysis are largely bespoke for each vehicle type in the trial.

\subsection{Soft Data Analysis}

In parallel with the hard data collection from the vehicles and charging infrastructure, SwitchEV also collects soft data from the trial participants. This includes a pre-drive survey, a survey and participation in focus groups about half way through the trail period and again when the triallists have completed their period with the vehicle. To date 172 pre-trail questionnaires have been completed and 68 post-trial. The soft data analysis collects information on the triallists opinions of electric vehicles and how this may change throughout the trial, how driving habits may change and when and how they charge their vehicles, as well as identifying any barriers to the wider adoption of electric vehicles. Where appropriate this data is correlated with the vehicle data loggers and charging event data.

\subsection{Server and Data Analysis}

The data is transferred to a server at Newcastle University using GPRS, when the ignition state switches from on to off, or off to on. Typically this means that the data from a single trip (where the ignition was on) would be transmitted at the end of the trip and the data from a charging event would be transmitted at the start of a trip. Additional data regarding the real-time road traffic flow and levels of congestion and environmental conditions are also collected and correlated with the in-vehicle data from the environmental motes developed under the MESSAGE project [5] and traffic flow and congestion data from the regional UTMC(Urban Traffic Management and Control Centre [6 and 7], as illustrated in figure 2.

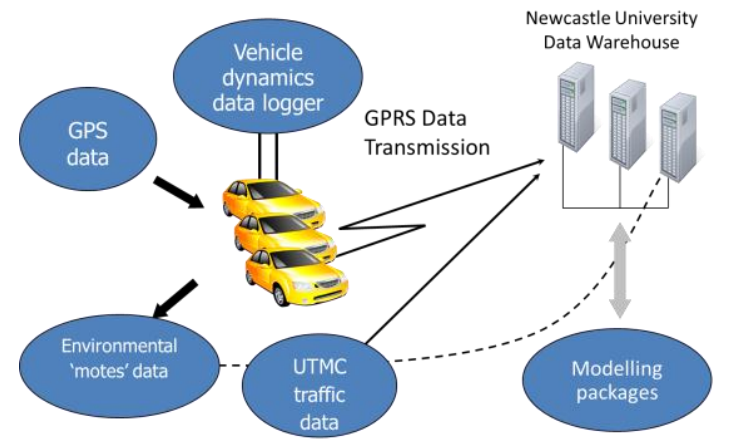

Figure 2: The ITS Infrastructure developed for the electric vehicle trial
To save the bandwidth when transmitting the data a simple raw byte format is used. Each time period measured is encoded in the raw byte format then concatenated. For example the first two bytes of the transmitted data indicated the logger ID number; the next six indicate the date, then GPS data, etc. After the data for a single time period has been sent, the system repeats. Although this format saves space within the data stream it is not a fault tolerant coding system hence it is necessary, after transmission, to fault check all data. If any errors in the data stream are flagged up then either the entire segment of data for that particular time period is discarded or of possible it is repaired.

A series of automated routines to format, repair and analyse the data elements were developed for the trial and implemented using the ' $R$ ' statistical analysis package. The detail of these routines will not be specifically described in the paper, however presentation of the results clearly illustrates some of the analysis routines developed.

The infrastructure and analysis packaged developed and deployed for the Switch-EV trial have now been running for 18 months and have proven robust (apart from a few logger hardware and software issues). The infrastructure platform developed for the SwitchEV project will operate until May 2013 for that specific project - but is now also being used to support several other industry, government and EU funded electric vehicle projects.

\section{Trial Results}

\subsection{Overview}

SwitchEV is recording the performance of the vehicle, the charging patterns (and locations) of the driver and, through questionnaires and focus groups, softer data: on attitudes, behaviour and other related information. Key to the demonstration programme are the following unknowns that are to be addressed by the trial:

- What will be the rate/extent of public adoption;

- What are the barriers to future adoption;

- Will driver behaviour vary:

- Range concern and use of vehicle;

- Re-charging preferences; and

- Vehicle performance?

- What is the real impact on $\mathrm{CO}_{2}$ emissions from EV's; 
- $\quad$ Strategic placement of charging posts and potential for multi-use of infrastructure.

The data for the analysis uses the SwitchEV trial data from December 2010 to January 2012, in that period the following overall summary of trial includes:

- $\quad$ Average journey length: $9 \mathrm{~km}$

- Longest journey: $136 \mathrm{~km}$

- Average journey duration: $15 \mathrm{~min}$

- Total journey distance: $\quad 277,852 \mathrm{~km}$

- $\quad$ Number of drive events: $\quad 37,910$

- Number of re-charge events: 13,863

- Total energy transferred: $48 \mathrm{MWhr}$

A selection of trial results

\subsection{A Selection of Trial Findings}

\subsubsection{Motivation for joining trial}

To understand the motivation why individuals and companies have leased electric vehicles and joined the SwitchEV trail some pre-trial questions relating to ascertaining why triallists signed up for the trial were given to 172 participants. Figure 3 shows that driving an EV so they can judge the vehicles performance themselves and a desire to do something to protect the environment were the two most cited reasons - this is not inconsistent with responses from other trials.

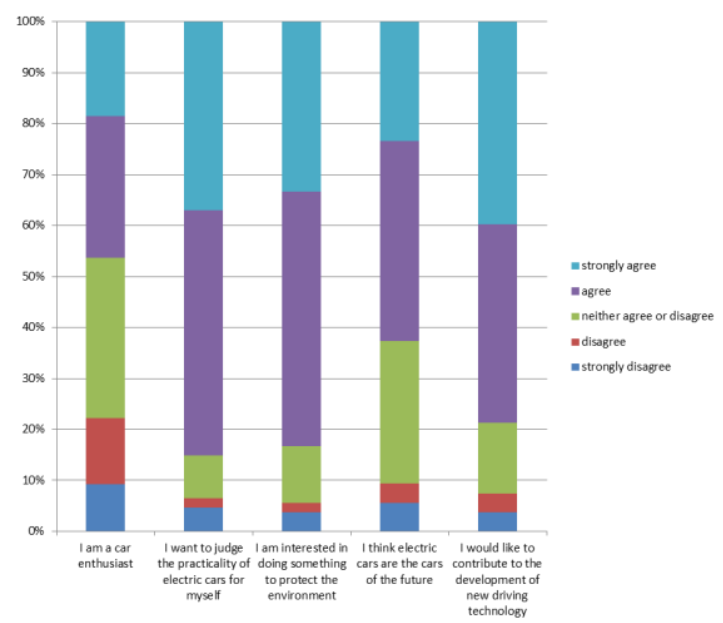

Figure 3: Respondents motivations to participating in Switch-EV trial

\subsubsection{Speed and range analysis}

Examining the usage of the electric vehicle and the utility that the triallists get out of the electric vehicle compared to the national figures for the $\mathrm{UK}$, the Switch-EV journeys were compared to the national average for trip data for all cars provided for the UK by the Department for Transport [8]. Figure 4 shows the SwitchEV drivers trip measured lengths in blue and the DfT figures in red. This illustrates that for urban driving the usage profile of the EV's is close in correlation to that of the general fleet

Utilising multi-month data for the fleet of 44 Switch-EV vehicles

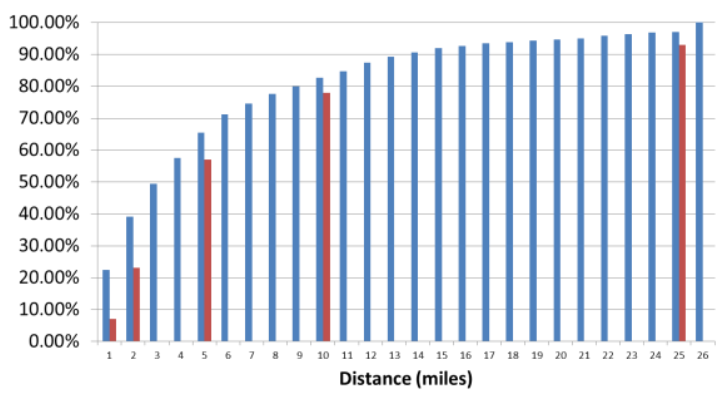

Figure 4: Measured trip length as a proportion of total trips made (EV Blue and DfT average Red).

Interestingly, soft data from the pre-trial questionnaire indicated that the participants overestimated the lengths of journeys they undertake with their car, with, for example for journey lengths under $5 \mathrm{~km}$ the pre-triallists suggested that was only about $2 \%$ of their journeys, whereas in reality the actually measured number of these short trips undertaken whilst on the trial was around $15 \%$ as reported in figure 5 .

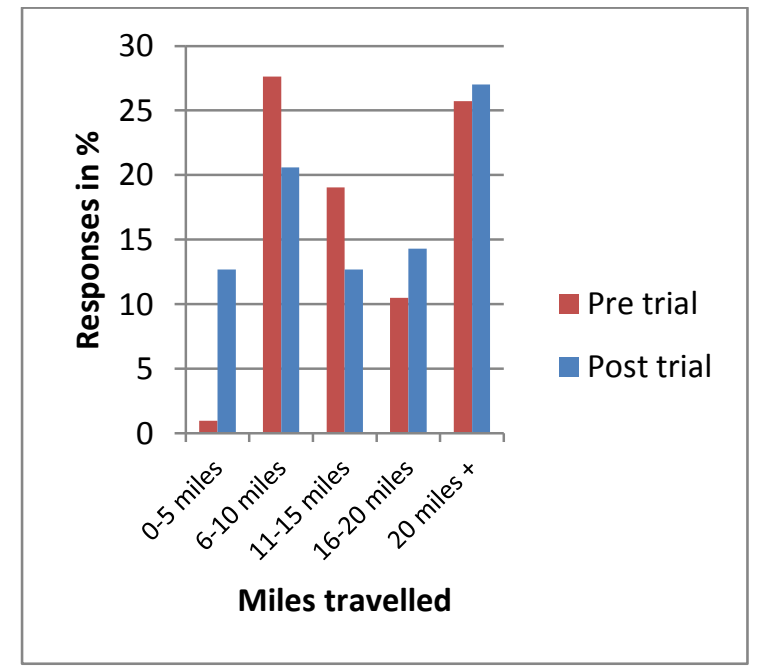

Figure 5: Estimate journey lengths (pre-trial) and measured journey lengths during trial

This figure is of significance as it may suggest that EV's are more suited for many drivers than they 
actually perceive as many journeys they regularly undertake are of shorter duration than they actually estimate.

The average speed distribution of trips was also measured by averaging each vehicles speed over a minute. This showed, as one would expect for urban driving a relatively low average speed (figure 6). Whilst figure 7 charts the peak speed recorded for a journey, for drivers who had just taken ownership of an EV for the trial (April 2011) and again for the same cohort of vehicles in June 2011 when the triallists were more familiar with the vehicle - this showed a $7 \%$ increase in peak speeds over the three months, suggesting that once drivers are familiar with the vehicle they become more willing to use the full performance of the vehicles (figure 7).

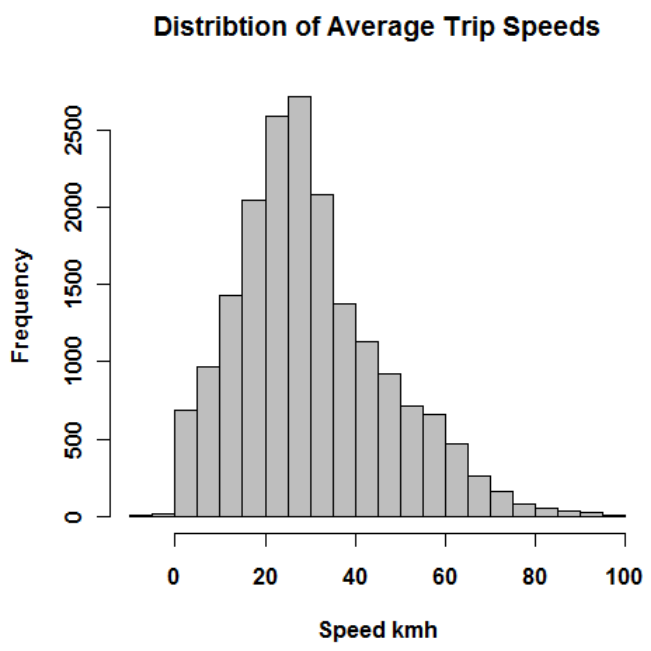

Figure 6: Average trip speeds for cohort \#1 drivers

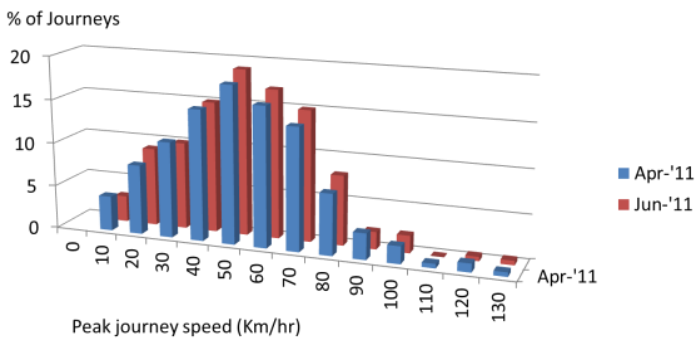

Figure 7: Comparison of peak journey speeds for triallists in April and June 2011

It was also found from both the trail data and from the soft data that drivers did tend to drive slightly slower in their EV's than they would in an ICE car - respondents suggested that this was partially to conserve battery life at high speeds and secondly they felt more responsible and 'enjoyed' driving the car more responsibility. Further research is underway to examine this hypothesis.

\subsubsection{Driving and trip analysis}

The in vehicle data logger provides a GPS based track of each EV's individual journey with speed, location, altitude and battery data recorded second by second. To illustrate the capability of the analysis tool: figure 8a shows a typical journey with GPS coordinates, superimposed with battery consumption and regeneration for the same journey; and figure 8b displays the same journey spatially with altitude to help research the relationship between road topology and battery usage/regeneration.

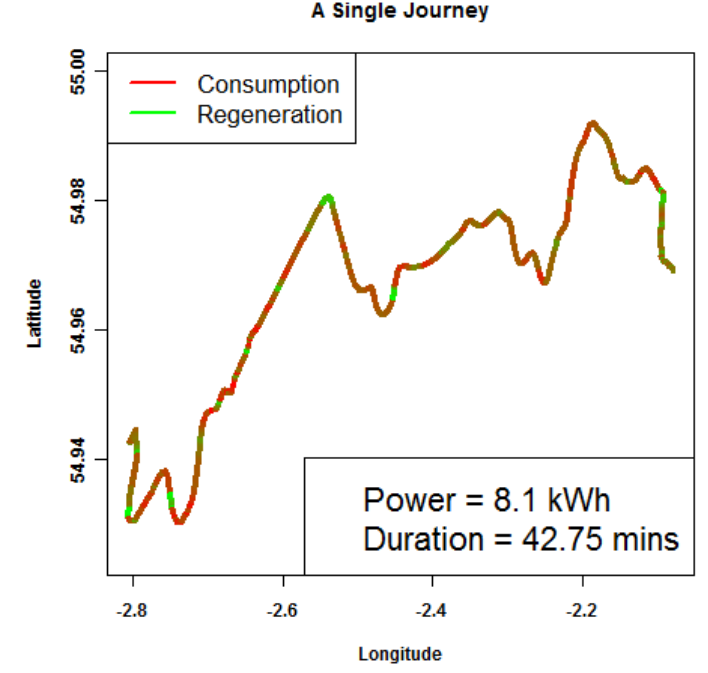

Figure 8a: Trip data with battery usage summary

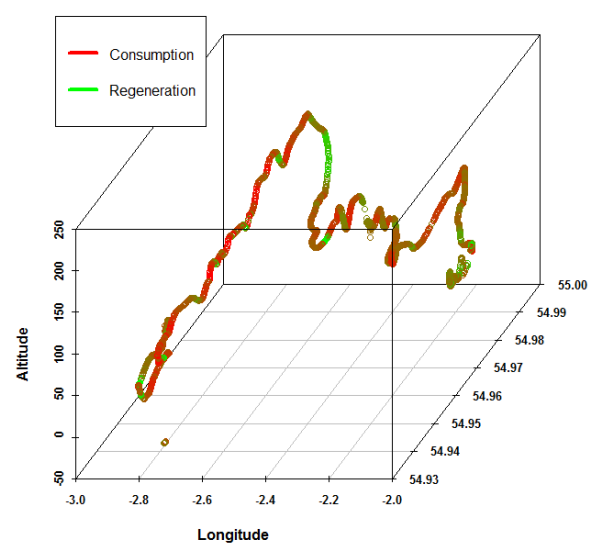

Figure 8b: Trip summary with topology features

The information on the battery consumption over a journey due to driving style, speed, road topology is also correlated with the levels of traffic 
congestion on certain roads (where the regional UTMC centre has traffic sensors) and with recorded weather conditions. Bringing all this data together is facilitating the development of a smart range estimator for the EV's which does not just consider the level of power remaining in the EV battery to estimate range but also features such inclines on roads on the desired routes, effects of temperature and expected levels of traffic (and congestion) on the planned route.

Further analysis of all of these features is underway to assist with the understanding of EV performance and their comparison to ICE vehicles. Utilising some early results from the developing model and comparing this to measured $\mathrm{CO} 2$ emissions from an instrumented ICE vehicle, figure 9 where using the speed and acceleration from the IC it is possible to simulate the $\mathrm{CO}_{2}$ emissions of an EV and compare.
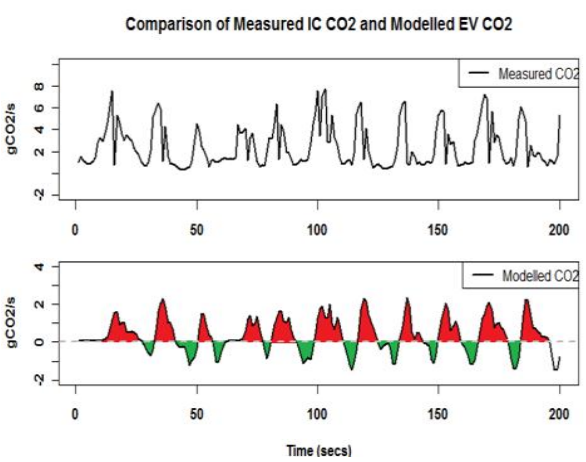

Figure 9: Measured $\mathrm{CO}_{2}$ emissions from an ICE vehicle and modelled emissions for the same journey for an UV vehicle.

\subsubsection{Re-charging behaviour}

Of particular interests to many regional and national players in the trial is re-charging behaviour as it will affect the policy on strategy on installing public charging posts, any government incentives to co-finance public, home and workplace charging posts and the future demand for electricity supply in different parts of the network and at different times of day.

To examine this the frequency, time, location and quantity of energy transferred at recharging events was recorded, as was the state of charge of the vehicles battery before the vehicle was recharged. The distribution of state of charge of a vehicle was used to estimate whether the triallists was 'brave', 'moderate' or 'cautious' in their attitudes to re-charging, figure 10a illustrates a typical distribution of charge for a 'brave' triallists and figure $10 \mathrm{~b}$ for a 'cautious' triallists.

Distribution of Car A Charging

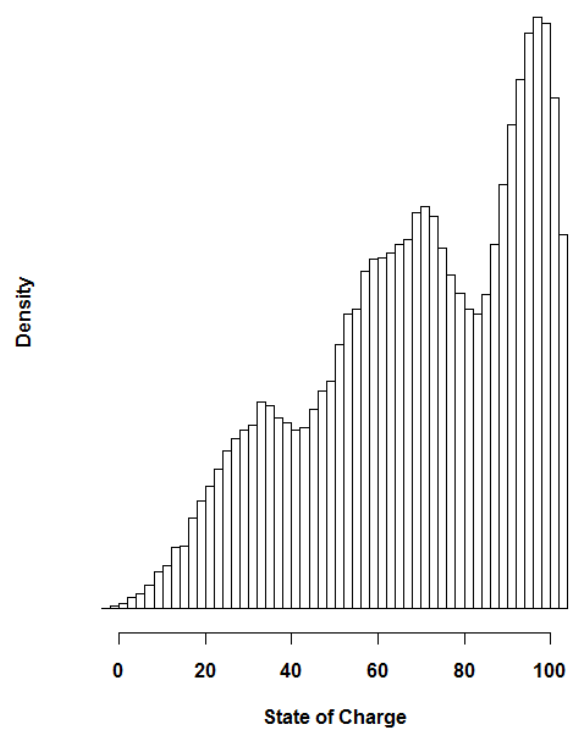

Figure 10a: A 'brave' charging profile

These measured traits were then correlated with the users own perception of their charging behaviour and tended to correlate well. Such correlations were not possible for pool-fleet electric vehicles where a number of users had access to the vehicle with different profiles.

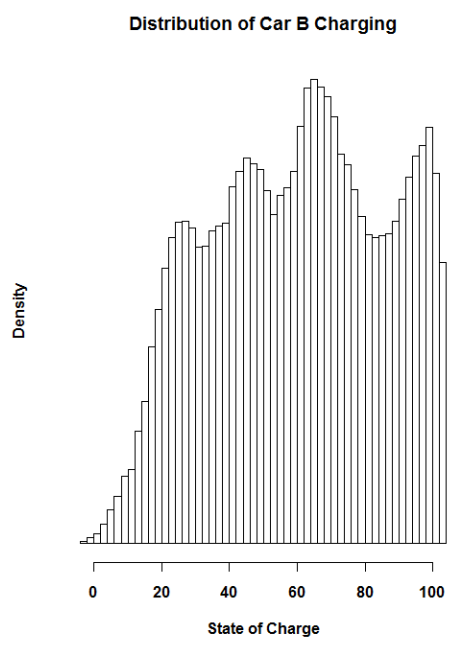

Figure 10b: A 'cautious' charging profile

A key tenant of the trial is to understand users recharging behaviour to better inform policy makers and planners where would be the most effective and optimum locations to install future charging posts and also what use of public infrastructure is 
made. As part of the TSB contractual requirements, every charging event recorded should be classified as 'home', 'work' or 'other'. To combine this data with the Charge your Car (CYC) infrastructure data we have sub-divided 'work' and 'other' to include CYC and non-CYC location charging events. The location of these events is mapped on figure 11 below:

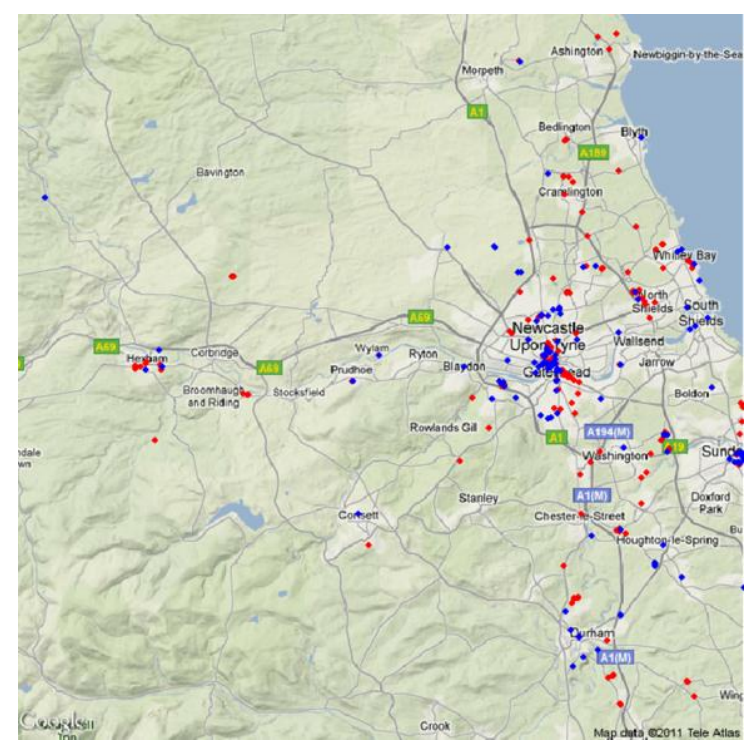

Figure 11: A map of recorded charging event locations (March to November 2011)

A surprising result was that almost $12 \%$ of charging events recorded were non-CYC other in other words locations the research team has no knowledge of. One may assume many of these may be where users have plugged their vehicles into standard electricity sockets, rather than installed charging points. This may have a significant impact on both electrical safety and demands on local electricity grids - hence significant work is currently on-going to understand this phenomenon further and quantify the implications of this behaviour [ 9 and 10].

To examine the effectiveness of the current spread of locations of publically available recharging posts in the NE of England - the research team recorded the journeys of thirty three of the electric vehicles on one specific day. From this we examined on a minute by minute basis the distance each of the vehicles were from the nearest available public charging post. This was a snapshot of journeys on one randomly selected day - and showed that for almost 90\% of the time the vehicles were within $5 \mathrm{~km}$ of a charging post and very rarely $(<1 \%)$ more than $20 \mathrm{~km}$ away, as shown in figure 12 . To date this analysis has only been performed for one day due to the computational challenges with analysing such data. This will improve over time.

Typical Distance to Nearest Charge Point

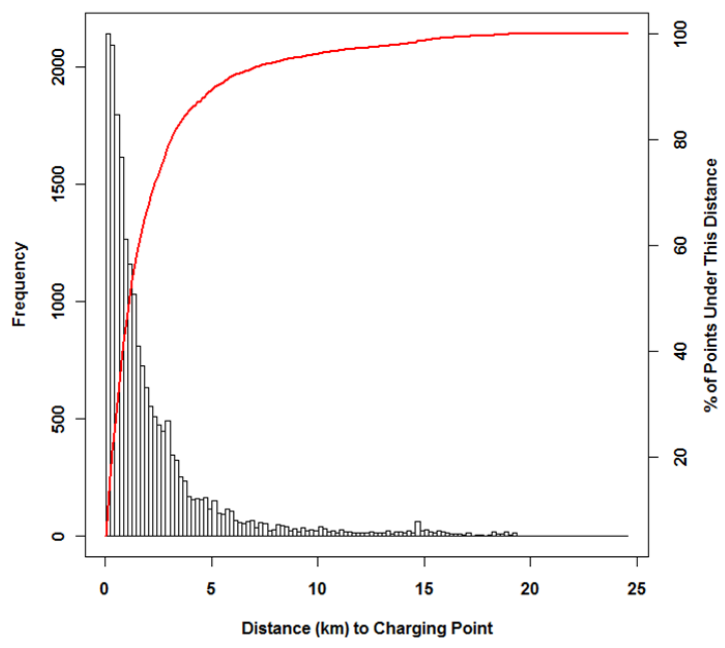

Figure 12: Shows vehicle distance from nearest charging post for a random selection of vehicles and journeys

Finally, in the post-trial questionnaires and focus groups it was confirmed that for the cohort of drivers in the SwitchEV trial, by far the most important location for charging vehicles was at work - where they perceived the electricity as free, figure 13.

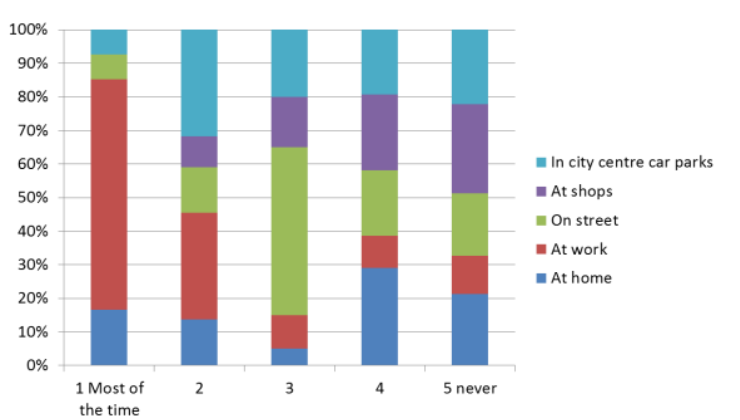

Figure 13: Triallists views on their most frequent recharging location

This result may be skewed by the large number of businesses recruited for the trial. However the location of the charging and the fact most of these charging events occur during daytime 'working hours' has implications for electricity supply and grid capabilities as this period is when the highest demand for electricity exists at the moment and it is also the period that has the highest level of $\mathrm{CO}_{2}$ in a kWhr of electricity supplied. Separate studies on this challenge are underway and one is presented in a separate paper at this conference [11]. 


\section{Summary}

This paper has provided an insight into the trial of 44 electric vehicles, currently underway in the North East of England. The trial itself is relatively unique in the sense the trial is utilising largely production model electric vehicles (35 of the 44) and the most dense network of EV recharging points in the UK. This gives the researchers and opportunity to begin to understand how drivers will use their vehicles in a 'real-world' situation and what re-charging activity will be established. This clearly will help to inform future planning and policy on the best locations for future charging posts to be sited and through extrapolation, an estimate of what implication the successful introduction of electric vehicles will have on household electricity demand and use, the local distribution grid network and future electricity demand and distribution in the UK.

Results from the trial suggest that although vehicles have limited range of around 80-90 miles, within that envelope the triallists have used their electric vehicles as they would their ICE's ones. Some evidence of driving slightly slower and safer has been suggested by both the hard data collected from the loggers and from interview and questionnaires.

Recharging behaviour suggest that the user will try to use 'free' electricity at work, or from posts provided under the CYC scheme and at the moment take little regard of the fact they are using more carbon-intensive 'peak' electricity rather than charging off-peak at home when the average $\mathrm{CO}_{2}$ embedded in the grids electricity is about $100 \mathrm{~g} / \mathrm{kWhr}$ less than at the peak.

Extensive work on both smart grids and smart electricity meters in both private homes and businesses is being addressed in the UK to begin to deal with this, indeed the last cohort of triallists in the SwitchEV project will each have a smart meter installed in their residence.

The SwitchEV trial will continue until May 2013 and a detailed set of research objectives have been defined to utilise the data logging, analysis and trial facilities to explore further insights in the use of electric vehicles and their associated re-charging network.

\section{Acknowledgments}

The authors gratefully acknowledge the funding support of the Technology Strategy Board and ONE North East and the support of all the SwitchEV partners in the project: Nissan, Simon Bailes Peugeot, Avid Vehicles, Smiths Electric Vehicles, Liberty Electric Vehicles, Charge Your Car/Low Carbon Futures and Future Transport Systems.

\section{References}

[1]

http://www.innovateuk.org/ourstrategy/innov ationplatforms/lowcarbonvehicles/ultralowcar bonvehicledemonstrator.ashx, accessed 0402-2012

[2] Hill, G., Blythe, P.T., and Suresh, V., (2010) How does the use of a continuously updating database allow for the analysis of a user's changing behaviour in electric vehicles? Proc. Road Traffic Information and Control, The IET, London June

[2] Hill, G., Blythe, P.T., and Suresh, V., (2010) How does the use of a continuously updating database allow for the analysis of a user's changing behaviour in electric vehicles? Proc. Road Traffic Information and Control, The IET, London June

[3] Blythe, P.T. (2011) ITS for Eco Driving and Carbon Reduction. Traffex Seminar on Low Carbon Transport, NEC Birmingham, 29th March

[4] www.chargeyourcar.org.uk/, accessed on 0502-2012

[5] North RJ., et al (2009) Field deployment of the MESSAGE System for environmental monitoring. Traffic Engineering \& Control 2009, 50(11), 480-483.

[6] Bell, M.C., et al..: 'UTMC Compliant Database to Support Technologies of the Future', Proc. IET Road Traffic Information and Control Conference, Manchester, May 2008

[7] King, R.: 'The Tyne and Wear UTMC System.' Proc. ITS on Your Doorstep.. ITS United Kingdom Seminar. Newcastle University, July 2011

[8] Department For Transport. (2011). Making the Connection: the Plug-In Vehicle Infrastructure Strategy. 1st June 2011. London:OLEV 
[9] Robinson, A., and Blythe, P.T. (2011) Application of ITS to maximise the efficiency of Electric Vehicles. Proc. HEVC, Cenex, Warwick, May

[10] Department For Business and Regulatory Reform. (2008). Investigation into the Scope for the Transport Sector to Switch to Electric Vehicles and Plug-in Hybrid Vehicles. London: HMSO

[11] Huebner, Y, et al (2012) Investigating the Potential to Influence the Behaviour of Electric Vehicle Users' Recharging Behaviour to Reduce Well to Wheel Carbon Emissions, Proc. EVS26, Los Angeles, May

${ }^{\mathrm{i}}$ OLEV is the Office of Low Emission Vehicles, an
agency of the UK Department for Transport (DfT) 